\title{
Post-growth thermal treatment of the InAs/GaAs quantum dots
}

\author{
J. Jasinski*, A. Babinski, R.Bozek, J. M. Baranowski \\ Institute of Experimental Physics, Warsaw University, \\ $69 \mathrm{Ho}_{\mathrm{i}}$ a St, 00-681 Warsaw, Poland \\ *also at Materials Science Division, Lawrence Berkeley National Laboratory, \\ 1 Cyclotron Rd, Berkeley CA 94720
}

\begin{abstract}
The effect of postgrowth thermal treatment of the InAs/GaAs quantum dots is investigated in this work. The photoluminescence (PL) and transmission electron microscopy (TEM) studies of samples annealed at temperatures up to $950^{\circ} \mathrm{C}$ are presented. A complete dissolution of QDs and substantial broadening of the wetting layer (WL) can be seen from TEM. We propose that the thermally induced modification of the WL rather than QDs can be responsible for a blue-shift and narrowing of $P L$ peaks in structures containing InAs/GaAs QDs.
\end{abstract}

\section{A. Introduction}

Thermally induced intermixing of semiconductor low-dimensional structures attracts an attention as a way of tuning their properties (for a review on quantum well intermixing see [1]. In recent years this technique has been applied to semiconductor quantum dots (QDs), which appear to be very promising in many optoelectronic applications [2]. Although there are many reports dealing with this problem, still some questions remain. In particular there is a debate over the character of carrier confinement in the intermixed QDs. In this study we will show that the thermal annealing of QDs at relatively high temperatures can lead to their decomposition and that photoluminescence (PL) blue-shift and narrowing in such structures can be due to the wetting layer (WL) modification.

\section{B. Experimental procedure}

Investigated structures were grown by metal organic vapour phase epitaxy (MOVPE) on GaAs (001) substrate. The InAs self-assembled QDs were grown in a Stranski-Krastanow growth mode at $470^{\circ} \mathrm{C}$ and they were overgrown by $100 \mathrm{~nm}$ thick GaAs layer. Four samples of this structure were annealed in rapid thermal annealing (RTA) system under nitrogen ambient for 30 seconds at $800^{\circ} \mathrm{C}, 850^{\circ} \mathrm{C}, 900^{\circ} \mathrm{C}$ and $950^{\circ} \mathrm{C}$. PL spectra of as-grown and annealed samples were measured at liquid helium temperature. Measurements were performed in a continuos flow CF-1204 Oxford cryostat with excitation with a semiconductor laser $(\lambda=780 \mathrm{~nm})$. The signal was dispersed by a $0.5 \mathrm{~m}$ monochromator and collected using a $\mathrm{LN}_{2}$ cooled germanium detector.

Transmission electron microscopy (TEM) was applied to study changes of sample microstructure. Cross-sectional TEM specimens were prepared from all investigated samples using standard mechanical thinning followed by ion milling. In addition, plan-view TEM specimen was prepared from as-grown sample by wet etching in 5\% methanol solution of $\mathrm{Br}$. 


\section{Photoluminescence}

PL spectrum of as grown sample consists of two distinct peaks (see Fig.1). A broad PL peak seen at $1.155 \mathrm{eV}$ is due to the optical recombination within the InAs QDs. Its broadening reflects an inhomogeneous broadening of QDs sizes. Other distinct PL feature can be observed at $1.42 \mathrm{eV}$, which is due to a thin InAs WL. It is known that both the QDs and WL PL peaks can be simultaneously observed at low QDs density [3]. Third broad PL feature observed in as grown structure is presumably due to WL regions of larger thickness. On the contrary to as-grown sample, only one PL peak can be observed in any of the annealed samples. The PL peaks in those samples are located at energies from $1.388 \mathrm{eV}\left(800^{\circ} \mathrm{C}\right)$ to $1.427 \mathrm{eV}\left(950^{\circ} \mathrm{C}\right)$. It can be seen in Fig.1 that higher annealing temperature the higher PL peak energy. The PL peak in annealed samples narrows with increasing annealing temperature. The PL intensity also increases with increasing RTA temperature.

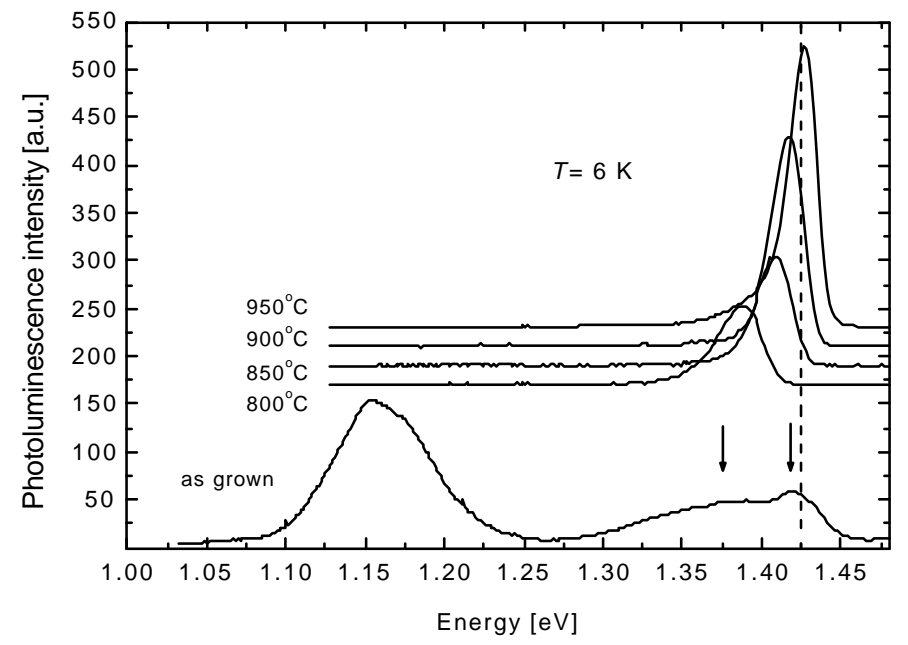

Fig.1. Low temperature photoluminescence from as grown and annealed samples. Photoluminescence from wetting layer is denoted with arrows.

The PL blue-shift and narrowing was previously reported for similar InAs QDs grown by molecular beam epitaxy [4]. However energy position of PL peaks in our annealed samples are significantly larger than reported previously. In that report, he PL from sample annealed at $800^{\circ} \mathrm{C}$ for $30 \mathrm{sec}$ peaks at $1.28 \mathrm{eV}$, the PL from sample annealed at $950^{\circ} \mathrm{C}$ for $30 \mathrm{sec}$ quenches and its excitation density behavior suggests the QDs -like attribution. This suggests that processes observed in our experiment are of different origin, which is probably due to differences between MOVPE and MBE growth conditions. We propose that two WL peaks in as grown sample are due to a thinner WL (presumably surrounding the QDs) and a thicker WL (in other regions). RTA treatment of our sample results in quenching of the PL from the thinner WL and from the QDs. This may be due to interdiffusion of In out of the QDs, which enriches surrounding WL regions. Simultaneously the thicker WL undergoes an intermixing, which results in a blue-shift and narrowing of its PL peak. Therefore in our experiment the PL seen from annealed structures is attributed to the WL and not to the QDs.

\section{Transmission Electron Microscopy}

TEM studies of as-grown sample confirmed the presence of self-assembled QDs in asgrown sample. Bright field TEM image of this sample taken under a two-beam $(0, \mathrm{~g})$ condition, where $\mathrm{g}$ was of the (220)-type reflection is shown on Fig.2. One can notice a high 
density of relatively small (with sizes in the range of $7 \div 10 \mathrm{~nm}$ ) QDs. They density estimated from plan-view images was $2 \times 10^{11} \mathrm{~cm}^{-2}$. We did not observed any big, dislocated islands or dislocations threading across GaAs capping layer in this sample.

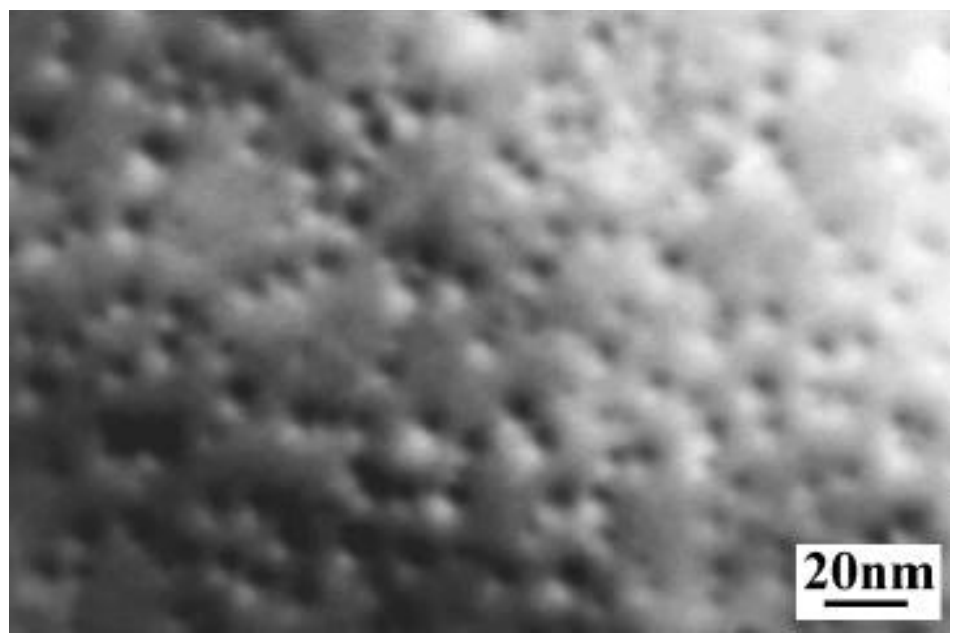

Fig.2. Bright field TEM image of the plan-view as-grown sample.

TEM results obtained for the plan-view of the as-grown sample are in agreement with the results of our high-resolution electron microscopy (HREM) studies of as-grown crosssectional specimen. Well-defined InAs QDs embedded between GaAs substrate and capping layer were visible on HREM images of this specimen (see Fig.3a).

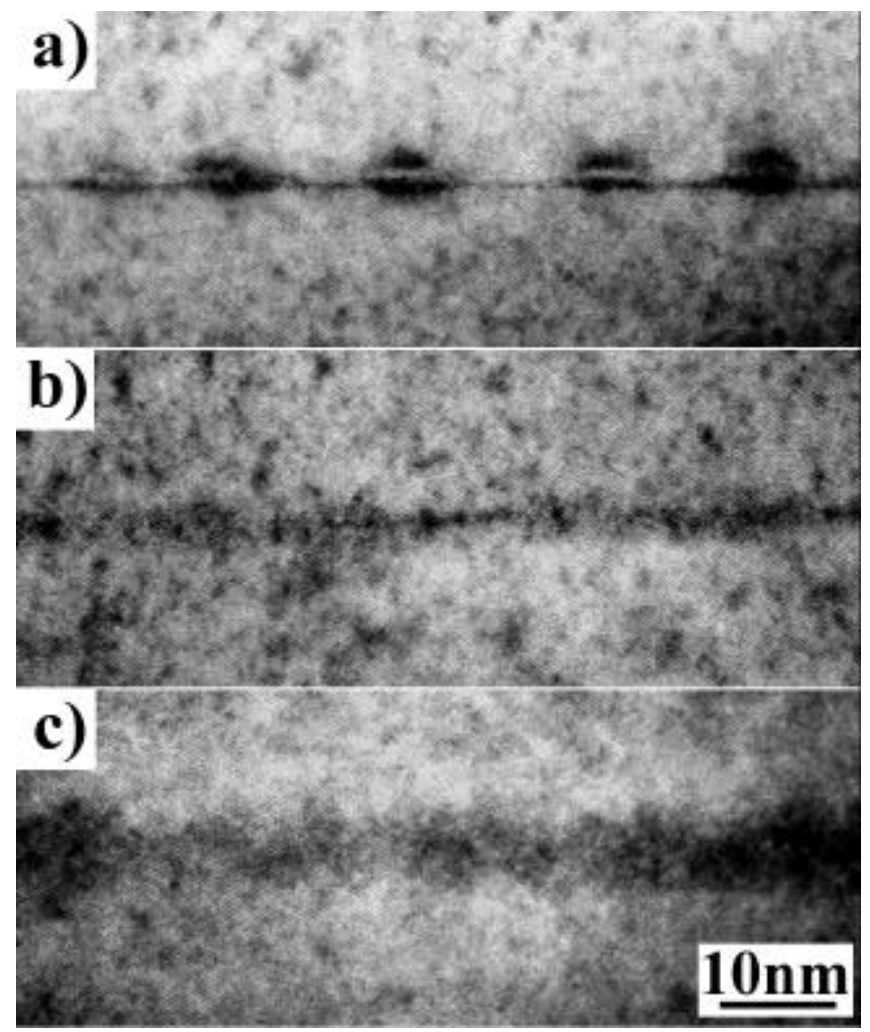

Fig.3. High resolution electron microscopy images of: as-grown (a), annealed at $800^{\circ} \mathrm{C}$ (b) and annealed at $950^{\circ} \mathrm{C}$ (c) samples. 
HREM images of as-grown sample in addition to InAs QDs provided also evidence of InAs wetting layer. This relatively thin layer with a thickness of approximately 2 monolayers, well visible in Fig.3a, connects adjacent QDs. In annealed samples this layer can be still visible however its thickness increases with the annealing temperature (see Fig.3b and Fig.3c). The layer thickness was found to increase up to approximately $3 \mathrm{~nm}$ after annealing at $800^{\circ} \mathrm{C}$ (Fig.3b) and up to about $10 \mathrm{~nm}$ after annealing at $950^{\circ} \mathrm{C}$ (Fig.3c). In addition to significant WL broadening and its interdiffusion RTA leads also to the drastic change of the layer morphology. No QDs can be seen in InAs layer after such thermal treatment. There are still some indium fluctuations visible in the HREM image of sample annealed at $800^{\circ} \mathrm{C}$ (Fig.3c), however these are not well defined QDs as in as-grown sample (Fig.3a).

\section{E. Conclusions}

In conclusion we studied an effect of thermal treatment on the structures with InAs/GaAs QDs grown by MOVPE. The results of PL and TEM studies of samples annealed at temperatures up to $950^{\circ} \mathrm{C}$ strongly suggest a complete dissolution of QDs and substantial broadening of the WL. It has been proposed that at least for high annealing temperatures thermally induced modification of the WL rather than QDs can be also responsible for a blueshift and narrowing of PL peaks in structures containing InAs/GaAs QDs.

\section{Acknowledgements}

This work was supported in part by the Polish Committee for Scientific Research Grant no. 7 T08 A 04017 and by U.S. Department of Energy under the Contract No. DE-AC0376SF00098. The use of the facility at the National Center for Electron Microscopy at Lawrence Berkeley National Laboratory is greatly appreciated.

\section{References}

[1] J. H. Marsh, Semic. Sc. Technol. 8 (1993) 1136.

[2] R. Leon, Yong Kim, C. Jagadish, M. Gal, J. Zou, and D. J. Cockayne, Appl. Phys. Lett. 69 (1996) 1888.

[3] R. Leon and S. Fafard, Phys. Rev. B 58 (1998) R1726.

[4] S. Malik, C. Roberts, R. Murray, M. Pate, Appl. Phys. Lett. 71, (1997) 1987. 